\title{
Metabolic Syndrome and Cardiovascular Risk after Liver Transplantation: A Single-Center Experience
}

\author{
M. Iadevaia, M. Giusto, V. Giannelli, Q. Lai, M. Rossi, P. Berloco, S.G. Corradini, and M. Merli
}

\begin{abstract}
Excessive weight gain, hypertension, hyperlipidemia, and diabetes are frequently observed among orthotopic liver transplantation (OLT) patients. These alterations, which are probably multifactorial in origin, contribute to posttransplantation metabolic syndrome (PTMS), which increases the risk of cardiovascular events. We assessed the prevalence of PTMS (diagnosed according to modified NCEP Adult Treatment Panel III criteria) in 156 OLT patients undergoing regular follow-up after transplantation (median 68 months; range, 6 to 234 months). Several pre- and post-OLT data were collected to identify the factors associated with the presence of PTMS which was found in $28 \%$ of cases. The only independent predictive factors for PTMS were diabetes mellitus and patients who were overweight or obese before-OLT. The prevalence of PTSM was lower among patients on tacrolimus immunosuppression. In our population, $21 \%$ of patients showed a high cardiovascular risk score with a $4 \%$ incidence of cardiovascular events, which was higher among subjects with PTMS. Close follow-up is mandatory to prevent the development of PTMS mainly among overweight and diabetic patients before transplantation.
\end{abstract}

B ECAUSE of improved survival after orthotopic liver transplantation (OLT), greater attention has been given to the diseases occurring in long-term follow-up. Metabolic syndrome (MS), as defined by National Cholesterol Education Program Adult Treatment Panel III criteria, ${ }^{1}$ is characterized by a cluster of manifestations including obesity, arterial hypertension, hyperglycemia, and dyslipidemia. After OLT, the prevalence of diabetes rises from $15 \%$ prior to OLT to $30-40 \%$ after transplantation, while hypertension increases from pre-transplant prevalence of $15 \%$ to $60-70 \%$ after transplantation (reference 2). The reported prevalence of dyslipidemia after transplantation is about $50-70 \% .^{2}$ Previous studies have reported posttransplant metabolic syndrome (PTMS) among $44 \%$ to $58 \%$ of patients after transplantation ${ }^{3,4}$ and is associated with an increased risk of cardiovascular and cerebrovascular events. ${ }^{5}$ Several predictors for PTMS have been identified as potential risk factors: higher age at transplant, male gender, pre-OLT diabetes/ obesity, the etiology of the underlying liver disease, and the choice of immunosuppressive agents. ${ }^{3-6}$ To improve survival among transplanted patients requires preventive measures, early recognition, and treatment of risk factors. We evaluated the prevalence and incidence of
PTMS to recognize risk factors for its development and to examine its association with cardiovascular risk.

\section{METHODS}

Adult stable-OLT recipients who were transplanted between 1990 and 2010 and continuously followed in our unit as outpatients were enrolled between April 2009 and August 2010. We excluded patients with low compliance, lack of clinical information, or less than 6 months follow-up. Demographic, lifestyle and socioeconomic data (age, sex, marital status, professional activity and education, smoking habits) and clinical data (time since OLT, indication for OLT, length of time on steroids after OLT, immunosuppressive therapy, arterial hypertension, and diabetes mellitus) were collected from our medical records. We also evaluated nutritional data before and during follow-up after OLT. PTMS was defined according to the modified NCEP Adult Treatment Panel III (ATPIII) criteria, wherein waist circumference was replaced with body mass index $>28.8$. For each patient we retrospectively

From the Department of Clinical Medicine, Division of Gastroenterology (M.I., M.G., V.G., S.G.C., M.M.), and the Department of Surgery "Paride Stefanini" (Q.L., M.R., P.B.), "Sapienza", University of Rome, Rome, Italy.

Address reprint requests to Manuela Merli, Dipartimento di Medicina Clinica, viale dell'Università 137, 00185 Rome, Italy. E-mail: manuela.merli@uniromal.it

0041-1345/-see front matter http://dx.doi.org/10.1016/j.transproceed.2012.06.022 
investigated time of appearance of each factor within the diagnosis of MS. Individual cardiovascular risk was also calculated using an Italian questionnaire available on www.cuore.iss.it. Student $t$ test as well as univariate and multivariate analyses were used to investigate the individual role of each risk factor to determine PTMS. A $P$ value $<.05$ was considered statistically significant.

\section{RESULTS}

We studied 156 patients including $76 \%$ males with an overall median age of 59 years. Their median time since transplantation was 68 months (range, 6 to 234 months). The main indications for liver transplantation were hepatitis C (38\%), hepatitis B (24\%), and alcohol abuse (22\%). Median time of steroid administration was 7 months (range, 1 to 24 months). Ninety-one percent of patients were treated with a calcineurin inhibitors (either cyclosporine or tacrolimus), while 9\% used sirolimus as primary immunosuppressant. Arterial hypertension and dyslipidemia were observed among 52\% and 58\% of patients, respectively, with diabetes in $30 \%$ and obesity in $20 \%$. The prevalence of PTMS was $28 \%$, including only $6 \%$ before OLT. The peak incidence for PTMS was observed within 1 year posttransplantation. Diabetes mellitus was the most relevant factor for the diagnosis of PTMS (95\%). Upon multivariate analysis, diabetes mellitus and overweight or obese before OLT were independent predictive factors for PTMS. The use of tacrolimus versus cyclosporine was associated with a lower incidence of PTMS (21\% versus $38 \%, P=$ .001). Among our study population, $27 \%$ of patients showed a high cardiovascular risk (78\% had PTMS). Cardiovascular events occurred in 6 patients $(4 \%$ of total), the majority of whom were men and belonged to the PTMS group (Fig 1).

\section{DISCUSSION}

This study revealed a $28 \%$ rate of PTMS among patients after liver transplantation, which was less than that reported in previous studies. ${ }^{3-6}$ Moreover, the prevalence of dyslipidemia, diabetes, and arterial hypertension was similar to that reported in other studies, but the rate of patients who were obese or overweight $(20 \%)$ was lower in our population. With regard to factors predicting PTMS, diabetes mellitus and patients who were overweight or obese before OLT were independent conditions. These conditions may help to achieve early identification of patients with an higher risk of post-OLT metabolic disturbances. In fact, the

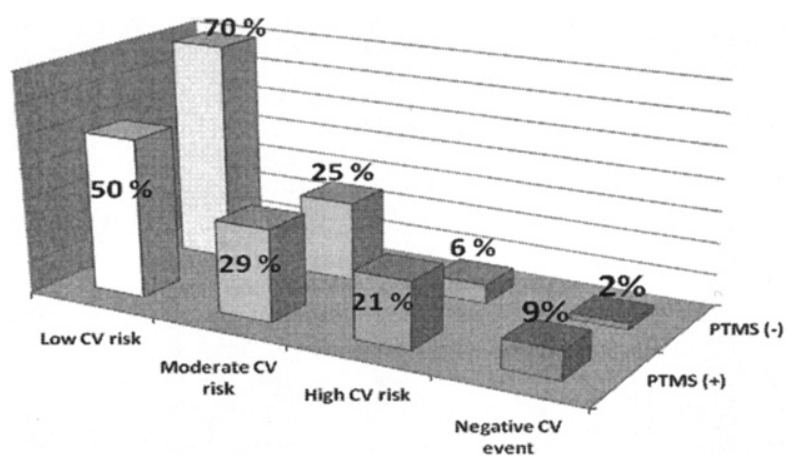

Fig 1. Cardiovascular (CV) risk scores in patients with and without posttransplantation metabolic syndrome (PTMS): Low CV risk, 0\% to 9.9\%; Moderate CV risk, $10 \%$ to $19.9 \%$; High CV risk, $\geq 20 \%$. (CV event represents cardiac or cerebral vascular event.)

majority of the patients developed PTMS during the first year after transplantation. At variance with another study, ${ }^{6}$ we failed to observe a correlation between hepatitis $C$ virus etiology and PTMS.

The clinical relevance of PTMS in liver transplant recipients is supported by the higher rate of cardiovascular event among this group. ${ }^{7}$ Four of 6 patients with a cardiovascular event in our population also had a diagnosis of PTMS.

\section{REFERENCES}

1. Ruiz-Rebollo ML, Sánchez-Antolin G, García-Pajares F, et al: Risk of development of the metabolic syndrome after orthotopic liver transplantation. Transplant Proc 42:663, 2010

2. Watt K, Pedersen RA, Kremers WK, et al: Evolution of causes and risk factors for mortality post liver transplant: results of the NIDDK long term follow up study. Am J Transplant 10:1420, 2010

3. Bianchi GP, Marchesini G, Marzocchi R, et al: Metabolic syndrome in liver transplantation: relation to etiology and immunosuppression. Liver Transp 4:1648, 2008

4. Francioso S, Angelico F, Baiocchi L, et al: High prevalence of metabolic syndrome and long-term survival after liver transplantation. J Hepatol 48:S82, 2008

5. Laryea M, Watt KD, Molinari M, et al: Metabolic syndrome in liver transplant recipients: prevalence and association with major vascular events. Liver Transpl 13:1109, 2007

6. Hanouneh IA, Feldstein AE, McCullough AI, et al: The significance of metabolic syndrome in the setting of recurrent hepatitis C after liver transplantation. Liver Transpl 14:1287, 2008

7. Mells G, Neuberger J: Reducing the risks of cardiovascular disease in liver allograft recipients. Transplantation 83:1141, 2007 